\title{
Prediction of superconductivity in pressure-induced new silicon boride phases
}

\author{
Xiaowei Liang $\odot,{ }^{1}$ Aitor Bergara, $, 2,3,4$ Yu Xie $\odot,{ }^{5, *}$ Linyan Wang, ${ }^{1}$ Rongxin Sun, ${ }^{1}$ Yufei Gao, ${ }^{1}$ Xiang-Feng Zhou, ${ }^{1}$ Bo Xu, ${ }^{1}$ \\ Julong He, ${ }^{1}$ Dongli Yu, ${ }^{1}$ Guoying Gao $\odot,{ }^{1, \dagger}$ and Yongjun Tian ${ }^{1}$ \\ ${ }^{1}$ Center for High Pressure Science, State Key Laboratory of Metastable Materials Science and Technology, \\ Yanshan University, Qinhuangdao 066004, China \\ ${ }^{2}$ Departmento de Física de la Materia Condensada, Universidad del País Vasco, UPV/EHU, 48080 Bilbao, Spain \\ ${ }^{3}$ Donostia International Physics Center (DIPC), 20018 Donostia, Spain \\ ${ }^{4}$ Centro de Física de Materiales CFM, Centro Mixto CSIC-UPV/EHU, 20018 Donostia, Spain \\ ${ }^{5}$ Key Laboratory of Physics and Technology for Advanced Batteries (Ministry of Education) \& Innovation Center for Computational Physics \\ Methods and Software \& State Key Laboratory of Superhard Materials, College of Physics, Jilin University, Changchun 130012, China
}

(Received 23 October 2019; published 27 January 2020)

\begin{abstract}
The crystal structures and properties of boron-silicon (B-Si) compounds under pressure have been systematically explored using particle swarm optimization structure prediction method in combination with first-principles calculations. Three new stoichiometries, $\mathrm{B}_{2} \mathrm{Si}, \mathrm{BSi}$, and $\mathrm{BSi}_{2}$, are predicted to be stable gradually under pressure, where increasing pressure favors the formation of silicon rich B-Si compounds. In the boron-rich compounds, the network of boron atoms changes from $B_{12}$ icosahedron in the ambient phases to the similar buckled graphenelike layers in the high-pressure phases, which crystalize in the same $P \overline{3} m 1$ symmetry but with different numbers of boron layers between adjacent silicon layers. Phonon calculations show that these structures might be retained to ambient conditions as metastable phases. Further electron-phonon coupling calculations indicate that the high-pressure phases of boron-rich compounds might superconduct at $1 \mathrm{~atm}$, with the highest $T_{c}$ value of $21 \mathrm{~K}$ from the Allen-Dynes equation in $P \overline{3} m 1 \mathrm{~B}_{2} \mathrm{Si}$, which is much higher than the one observed in boron doped diamond-type silicon. Moreover, further fully anisotropic Migdal-Eliashberg calculations indicate that $\mathrm{B}_{2} \mathrm{Si}$ is a two-gap anisotropic superconductor and the estimated $T_{c}$ might reach up to $30 \mathrm{~K}$ at $1 \mathrm{~atm}$. On the silicon-rich side, $\mathrm{BSi}_{2}$ is predicted to be stable in the $\mathrm{CuAl}_{2}$-type structure. Our current results significantly enrich the phase diagram of the B-Si system and will stimulate further experimental study.
\end{abstract}

DOI: 10.1103/PhysRevB.101.014112

\section{INTRODUCTION}

Borides have attracted much attention in both fundamental science and industrial applications owing to their potential superconducting, superhard, thermoelectric, and refractory properties [1-12]. These diverse properties are related to a wide variety of structures and boron arrangements in borides (ranging from isolated boron atoms to complex polyhedron) [1-3]. Like most of borides, the known silicon borides consisting of boron polyhedral frameworks have extremely high thermal and chemical stability in high corrosive and high temperature environments, thus they are typically refractory materials and often used in devices operating at extreme conditions [13-15]. Recently, boron-doped silicon has attracted considerable attention for its potential superconductivity [16-19] and its critical superconducting temperature rises up to $0.56 \mathrm{~K}$ with increasing the boron content [19].

The B-Si system has been the subject of extensive investigation since 1900 [20]. At ambient pressure and high temperature, three approximate compositions, $\mathrm{B}_{3} \mathrm{Si}$ [14,21-23], $\mathrm{B}_{6} \mathrm{Si}[24,25]$, and $\mathrm{B}_{n} \mathrm{Si}(n \geqslant 14)$ [26-28], have been observed in experiments. $\alpha-\mathrm{B}_{3} \mathrm{Si}$, with stoichiometries

\footnotetext{
*xieyu@jlu.edu.cn

†gaoguoying@ysu.edu.cn
}

ranging from $\mathrm{B}_{2.8} \mathrm{Si}$ to $\mathrm{B}_{4} \mathrm{Si}$, containing boron-rich icosahe$\mathrm{dra}$, was proposed to be rhombohedral, where silicon atoms partially substitute boron atoms in the polar icosahedral sites [22]. This compound forms from direct combination of the elements by isothermal heating at $1225^{\circ} \mathrm{C}$ or arc melting in a high-frequency induction furnace. The first fully crystallographically ordered structure, $\beta-\mathrm{B}_{3} \mathrm{Si}[14]$, was produced in Ga flux at a lower temperature $\left(850-1000^{\circ} \mathrm{C}\right)$, and adopts an orthorhombic structure with $\mathrm{B}$ atoms forming regular $\mathrm{B}_{12}$ icosahedra. Another stoichiometry with an approximate ratio of $\mathrm{B}: \mathrm{Si}=6: 1\left(\mathrm{~B}_{6} \mathrm{Si}\right)$ is also known, which contains boron-rich icosahedra, icosihexahedra, as well as isolated silicon and boron atoms. $\alpha-\mathrm{B}_{3} \mathrm{Si}, \beta-\mathrm{B}_{3} \mathrm{Si}$, and $\mathrm{B}_{6} \mathrm{Si}$ are all semiconductors with band gaps of $0.2,2.0$, and $1.2 \mathrm{eV}$, respectively $[14,15]$. As an extension of solid solution of silicon in boron, the structure of $\mathrm{B}_{n} \mathrm{Si}(n \geqslant 14)$ is similar to pure $\beta$ boron [26,27], with silicon atoms partially occupying icosahedral boron atom positions or interstitial sites. It is well known that the physical properties of a material are closely related to its crystal structures. The experimentally observed structures of $\mathrm{B}_{3} \mathrm{Si}$, $\mathrm{B}_{6} \mathrm{Si}$, and $\mathrm{B}_{n} \mathrm{Si}$ contain similar $\mathrm{B}$ polyhedral frameworks, but there is no report on other structural features formed by $\mathrm{B}$ atoms in boron-rich compounds yet. On the silicon-rich side, there is only one theoretical work on $\mathrm{BSi}_{3}$ by Wang et al. [29], where they predicted three metastable structures, $P 3{ }_{1} 21, C 2 / m$, and $P 21 / m$, in the pressure range of $0-160 \mathrm{GPa}$, 
which are potential hard materials. Small-size $\mathrm{B}_{n} \mathrm{Si}^{0 /-}$ clusters and two-dimensional boron-silicon compounds were also theoretically investigated [30-34].

Here, we have extensively investigated the crystal structures and properties of $\mathrm{B}-\mathrm{Si}$ compounds under pressure by using the particle swarm optimization structure prediction method in combination with first-principles calculations. We first explored the already known $\mathrm{B}_{6} \mathrm{Si}, \mathrm{B}_{4} \mathrm{Si}$, and $\mathrm{B}_{3} \mathrm{Si}$ stoichiometries and then proceed to the analysis of hypothetical $\mathrm{B}_{2} \mathrm{Si}, \mathrm{BSi}, \mathrm{BSi}_{2}$, and $\mathrm{BSi}_{3}$ stoichiometries. We studied in detail the crystal structures of the preferred compounds as a function of pressure, together with their dynamical stability and corresponding electronic band structures and superconductivity. We found that three new stoichiometries, $\mathrm{B}_{2} \mathrm{Si}, \mathrm{BSi}$, and $\mathrm{BSi}_{2}$, become stable gradually under pressure, where increasing pressure favors the formation of silicon rich $\mathrm{B}-\mathrm{Si}$ compounds. All the predicted stable high-pressure structures of boron-rich compounds have similar structural characteristics containing buckled graphenelike boron layers. In addition, our calculations indicate that these structures might be preserved to ambient pressure as metastable phases, in which they were predicted to be superconductors with the highest $T_{c}$ value of $30 \mathrm{~K}$ in $P \overline{3} \mathrm{~m} 1 \mathrm{~B}_{2} \mathrm{Si}$.

\section{COMPUTATIONAL DETAILS}

Structure searches of $\mathrm{B}_{x} \mathrm{Si}_{y} \quad(x=2-4$, and $6, y=1$; $x=1, y=1-3)$ were performed using the particle swarm optimization method [35,36], as implemented in the CALYPSO code [37]. Such an approach has successfully predicted the high-pressure structures of various systems, ranging from elemental solids to binary and ternary compounds [38-44]. The underlying energetic calculations were performed within the framework of density functional theory (DFT) as implemented in the VASP code [45]. The Perdew-BurkeErnzerhof generalized gradient approximation was chosen for the exchange-correlation functional [46]. The adopted allelectron projector-augmented wave (PAW) [47] pseudopotentials of $\mathrm{B}$ and $\mathrm{Si}$ treat $2 s^{2} 2 p^{1}$ and $3 s^{2} 3 p^{2}$ electrons as valence electrons, respectively. An energy cutoff of $700 \mathrm{eV}$ and appropriate Monkhorst-Pack $k$ meshes [48] were chosen to ensure that enthalpy calculations were well converged within $1 \mathrm{meV} / \mathrm{f}$.u. Phonon calculations were performed by the direct supercell method [49] or density functional perturbation theory (DFPT) with the PHONOPY [50] and QUANTUM-ESPRESSO codes [51], respectively. Electron-phonon coupling (EPC) calculations use the ultrasoft pseudopotential method within DFPT. Convergence tests concluded a kinetic energy cutoff of 60 Ry. $6 \times 6 \times 6,8 \times 8 \times 6,9 \times 9 \times 3$, and $7 \times 7 \times 4$ $q$-point meshes in the first Brillouin zone were used in the EPC calculation for $R \overline{3} m \mathrm{~B}_{2} \mathrm{Si}, P \overline{3} m 1 \mathrm{~B}_{2} \mathrm{Si}, P \overline{3} m 1 \quad \mathrm{~B}_{3} \mathrm{Si}$, and $P \overline{3} \mathrm{~m} 1 \mathrm{~B}_{4} \mathrm{Si}$, respectively. Correspondingly, Monkrost-Pack grids of $24 \times 24 \times 24,32 \times 32 \times 24,36 \times 36 \times 12$, and $28 \times$ $28 \times 16$ were used to ensure $k$-point sampling convergence with Gaussians of width $0.02 \mathrm{Ry}$. The superconducting gap of $P \overline{3} \mathrm{~m} 1 \mathrm{~B}_{2} \mathrm{Si}$ was estimated using the anisotropic MigdalEliashberg formalism [52,53] as implemented in the EPW code [54-56]. We used norm-conserving pseudopotentials with a plane-wave basis up to $60 \mathrm{Ry}$. The electron-phonon matrix elements were calculated on coarse phonon and electron grids of $7 \times 7 \times 5$ and $14 \times 14 \times 10$, respectively, and then interpolated onto fine $q$ and $k$ grids of $56 \times 56 \times 40$ and $56 \times 56 \times 40$ by Wannier-Fourier interpolation [57,58].

\section{RESULTS AND DISCUSSION}

The crystal structure searches of $\mathrm{B}_{x} \mathrm{Si}_{y}(x=2-4$, and 6 , $y=1 ; x=1, y=1-3)$ were carried out with system sizes containing up to 8 formula units (f.u.) per simulation cell over a wide pressure range of $0-400 \mathrm{GPa}$. Our structure prediction starts from the already known compounds at low pressure. For $\mathrm{B}_{3} \mathrm{Si}$, an orthorhombic Pmma structure was predicted to be stable against decomposition into elements (B and $\mathrm{Si}$ ). Its structure is very similar to the experimentally observed Imma $\beta$ - $\mathrm{B}_{3} \mathrm{Si}$ (Fig. S1 [59]). Both structures are composed of interlinked $\mathrm{B}_{12}$ cages and $\mathrm{Si}_{4}$ chains. However, there is a little difference in the relative positions of some $\mathrm{Si}$ atoms (atoms 2 and 4) [Fig. S1(a) [59]]. We made an estimation of the energy barrier between them by shifting $\mathrm{Si}$ atoms and the calculated maximum energy barrier reaches $0.12 \mathrm{eV} /$ f.u. at $0 \mathrm{~K}$ [Fig. S1(b) [59]], which prevents the transition from Pmma to Imma. However, this barrier can be easily overcome at high temperatures so that the two structures might be interchangeable.

For $\mathrm{B}_{4} \mathrm{Si}$ and $\mathrm{B}_{6} \mathrm{Si}$, a series of energetically competitive structures (Figs. S2-3 [59]) were predicted to contain B-rich cages, in agreement with the experimental observations. The predicted $R \overline{3} m \mathrm{~B}_{6} \mathrm{Si}$ is isomorphous with the well-known $R \overline{3} m$ $\mathrm{B}_{6} \mathrm{O}$ [60]. Cmcm $\mathrm{B}_{6} \mathrm{Si}$ is the most stable phase between 0 and 17.5 GPa and then transforms to a similar Cmca structure, which is the same with the Cmca $\mathrm{LiB}_{6} \mathrm{Si}$ getting rid of $\mathrm{Li}$ atoms [61]. Different from $\mathrm{B}_{3} \mathrm{Si}$, all the predicted structures for $\mathrm{B}_{4} \mathrm{Si}$ and $\mathrm{B}_{6} \mathrm{Si}$ are metastable with positive formation enthalpies, though the enthalpies of $P 2 / c \mathrm{~B}_{4} \mathrm{Si}$ are only 3$11 \mathrm{meV}$ higher than those of $4 \mathrm{~B}+\mathrm{Si}$ at the pressure range of 0-10 GPa. Notably, all the already known B-Si compounds, with the exception of $\beta$ - $\mathrm{B}_{3} \mathrm{Si}$, were observed to be disordered and the prediction of disordered structures goes beyond the scope of our research. However, the successful prediction of $\beta$ - $\mathrm{B}_{3} \mathrm{Si}$ and $\mathrm{B}_{4} \mathrm{Si}$ and $\mathrm{B}_{6} \mathrm{Si}$ cagelike structures confirms the reliability of our method.

To explore the possible new stoichiometries, we focused our structural searches on $\mathrm{B}_{x} \mathrm{Si}_{y}$ at $1 \mathrm{~atm}, 10,50,100$, 200, 300, and $400 \mathrm{GPa}$. The thermodynamic stabilities were evaluated by calculating their formation enthalpies relative to the products of dissociation into constituent elements. As references, the $R \overline{3} \mathrm{~m}, \mathrm{Pnnm}, \mathrm{Cmca}$, and $P 6_{3} / \mathrm{mcm}$ structures for $\mathrm{B}$ and $F d \overline{3} m, P 6_{3} / m c m$, and $F m \overline{3} m$ for $\mathrm{Si}$ are considered, each in its most stable form at the specified pressure [62-64]. To account for the enthalpy relationships of each stoichiometry, a convex hull for the B-Si system was calculated at different pressures, as depicted in Fig. 1(a). Formation enthalpies of compounds are located on the hull, showing they are thermodynamically stable with respect to elements or other binary compounds. At $50 \mathrm{GPa}$, the already known B-rich compounds are predicted to decompose into elemental $\mathrm{B}$ and $\mathrm{Si}$, and no other stoichiometries can be formed. With increasing pressure to $65 \mathrm{GPa}, \mathrm{B}_{2} \mathrm{Si}$, $\mathrm{B}_{3} \mathrm{Si}$, and $\mathrm{B}_{4} \mathrm{Si}$ become energetically favored over elemental 


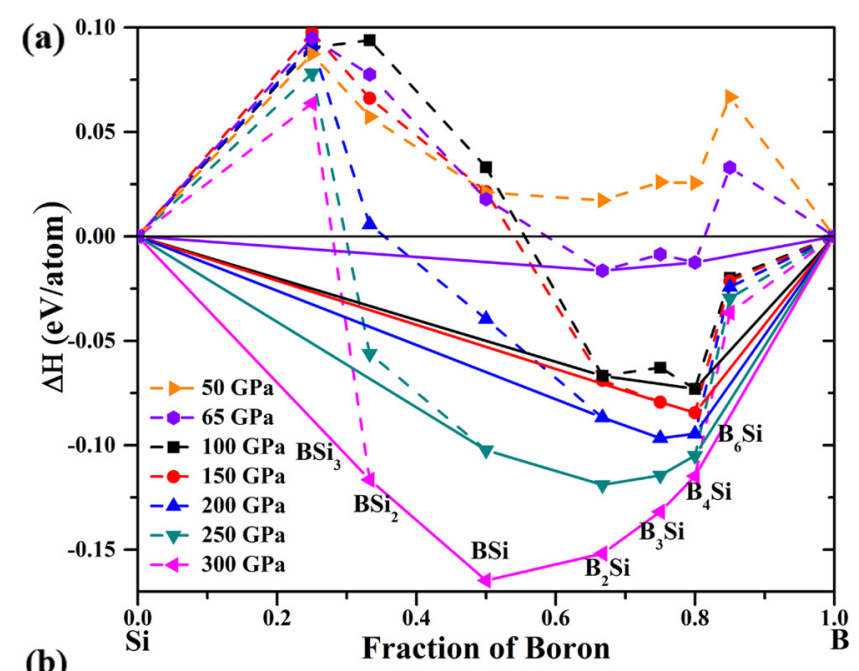

(b)

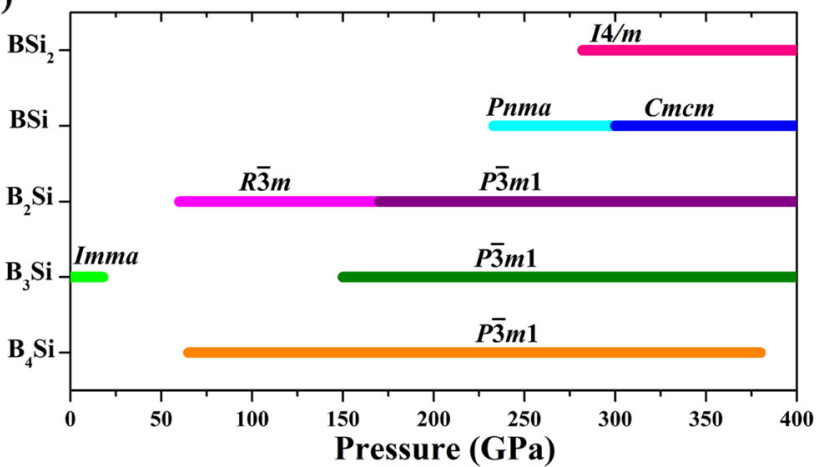

FIG. 1. (a) Formation enthalpies $(\Delta H)$ per atom of the $\mathrm{B}_{x} \mathrm{Si}_{y}$ phases with respect to their separated counterparts at 50-300 GPa. The symbols located on the solid lines indicate that the compound is stable at the corresponding pressure. (b) The predicted pressurecomposition phase diagram of the B-Si system.

dissociation, although $\mathrm{B}_{3} \mathrm{Si}$ is still unstable against decomposition into $\mathrm{B}_{2} \mathrm{Si}$ and $\mathrm{B}_{4} \mathrm{Si}$. As pressure increases to $150 \mathrm{GPa}$, the formation enthalpies of $\mathrm{B}_{2} \mathrm{Si}, \mathrm{B}_{3} \mathrm{Si}$, and $\mathrm{B}_{4} \mathrm{Si}$ become more and more negative (more stable) and eventually $\mathrm{B}_{3} \mathrm{Si}$ falls on the convex hull. Upon further compression, at 250 and $300 \mathrm{GPa}, \mathrm{BSi}$ and $\mathrm{BSi}_{2}$, respectively, gradually become stable with respect to disproportionation into other stoichiometries. Pressure clearly favors the formation of B-Si compounds with a high content of silicon. Interestingly, as pressure increases from 65 to $250 \mathrm{GPa}, \mathrm{B}_{2} \mathrm{Si}$ becomes easier to form. Similarly, pressure also plays a positive role in the formation of $\mathrm{BSi}$.

The predicted pressure-composition phase diagram and respective enthalpy curves of corresponding B-Si compounds are shown in Fig. 1(b) and Fig. S4 [59], respectively. An orthorhombic Pnma BSi is predicted to be stable between 233 and $300 \mathrm{GPa}$ and transforms into an energetically favored Cmcm structure above $300 \mathrm{GPa} . R \overline{3} m$ and $P \overline{3} \mathrm{~m} 1 \mathrm{~B}_{2} \mathrm{Si}$ are enthalpically stable at pressure ranges of $60-170 \mathrm{GPa}$ and 170-400 GPa, respectively. The experimentally observed $\beta$ $\mathrm{B}_{3} \mathrm{Si}$ and the similar theoretically predicted Pmma structure are stable up to $18 \mathrm{GPa}$. Note that $\mathrm{B}_{3} \mathrm{Si}$ decomposes into pure elements and other compounds at the pressure range of $18-150 \mathrm{GPa}$, while it becomes stable again with a $P \overline{3} \mathrm{~m} 1$ structure above $150 \mathrm{GPa}$. The $P \overline{3} m 1$ structure for $\mathrm{B}_{4} \mathrm{Si}$ is

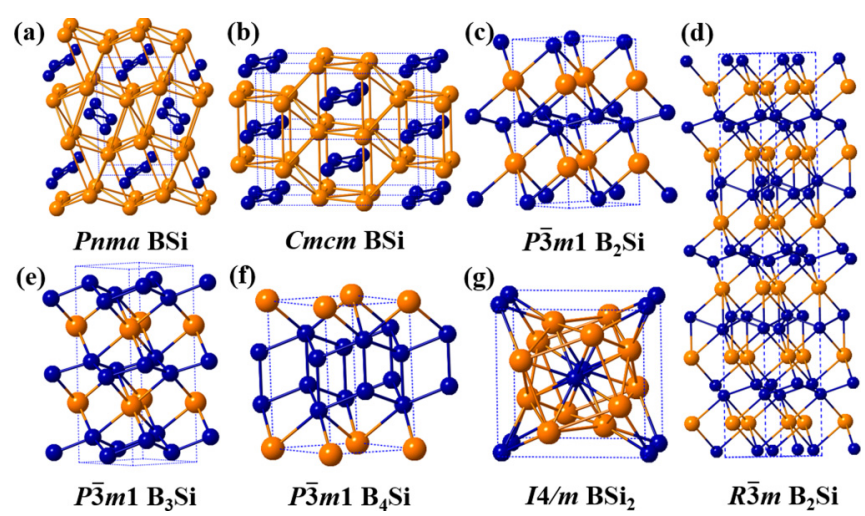

FIG. 2. Predicted stable crystal structures of studied B-Si compounds under pressure. (a) Pnma BSi at $250 \mathrm{GPa}$. (b) $\mathrm{Cmcm} \mathrm{BSi}$ at $350 \mathrm{GPa}$. (c) $P \overline{3} m 1 \mathrm{~B}_{2} \mathrm{Si}$ at $200 \mathrm{GPa}$. (d) $R \overline{3} m \mathrm{~B}_{2} \mathrm{Si}$ at $100 \mathrm{GPa}$. (e) $P \overline{3} m 1 \mathrm{~B}_{3} \mathrm{Si}$ at $150 \mathrm{GPa}$. (f) $P \overline{3} m 1 \mathrm{~B}_{4} \mathrm{Si}$ at $100 \mathrm{GPa}$. (g) $I 4 / m$ $\mathrm{BSi}_{2}$ at $300 \mathrm{GPa}$. Blue and orange spheres represent $\mathrm{B}$ and $\mathrm{Si}$ atoms, respectively.

stable over a wide pressure range of $65-380 \mathrm{GPa}$ and $I 4 / \mathrm{m}$ $\mathrm{BSi}_{2}$ will be stable at pressures between 282 and $400 \mathrm{GPa}$.

As a basic requirement for the structural stability, the dynamical stability of various structures for B-Si compounds is also examined. The phonon dispersion curves of all the predicted structures in the corresponding stable pressure ranges are presented in Fig. S13 [59]. The absence of any imaginary phonon frequency in the entire Brillouin zone shows they are dynamically stable. Interestingly, $R \overline{3} m$ and $P \overline{3} m 1 \mathrm{~B}_{2} \mathrm{Si}, C 2 / m$ and $P \overline{3} m 1 \quad \mathrm{~B}_{3} \mathrm{Si}$, and $P \overline{3} m 1 \mathrm{~B}_{4} \mathrm{Si}$ were also calculated to be dynamically stable at $1 \mathrm{~atm}$, which indicates that these predicted high-pressure phases might be also retained to ambient pressure.

The predicted stable structures are shown in Fig. 2. Both Pnma and $\mathrm{Cmcm}$ structures of BSi are orthorhombic and have the same configurations as the already known Pnma FeB and $\mathrm{Cmcm} \mathrm{CrB}$, respectively [2]. In both structures, B atoms form zigzag chains and $\mathrm{Si}$ atoms form a column of trigonal prisms arranging along the B-B chains. With increasing B content, the B-B bonding changes significantly. As shown in Figs. 2(c) and 2(d), the zigzag B-B chains in BSi transform to buckled six-membered rings in the predicted structures for $\mathrm{B}_{2} \mathrm{Si}$. The $P \overline{3} m 1 \mathrm{~B}_{2} \mathrm{Si}$ is similar to the structure of $\mathrm{MgB}_{2}$. Si atoms are located at edges of the unit cell and a deformed graphenelike boron layer is located between $\mathrm{Si}$ layers. The $R \overline{3} m \mathrm{~B}_{2} \mathrm{Si}$ can be considered a slight modification of the $P \overline{3} \mathrm{~m} 1$ structure, containing two types of boron atoms with coordination numbers of 6 and 7, respectively. Interestingly, the predicted stable high-pressure structures of $\mathrm{B}_{3} \mathrm{Si}$ and $\mathrm{B}_{4} \mathrm{Si}$ have similar geometries to that of $\mathrm{B}_{2} \mathrm{Si}$, which are crystalized in the same $P \overline{3} m 1$ symmetry. For $P \overline{3} m 1 \mathrm{~B}_{3} \mathrm{Si}$ and $\mathrm{B}_{4} \mathrm{Si}$, B atoms also exist in the form of buckled graphenelike layers [65]. The main difference between $P \overline{3} m 1 \mathrm{~B}_{2} \mathrm{Si}, \mathrm{B}_{3} \mathrm{Si}$, and $\mathrm{B}_{4} \mathrm{Si}$ is the number of boron layers between adjacent silicon layers, which increases with increasing the boron content. However, as boron content increases to $\mathrm{B}_{6} \mathrm{Si}$, where the predicted structures present more than three boron layers between silicon layers (Fig. S5 [59]), it is unstable over the whole studied pressure range with respect to decomposing into $\mathrm{B}_{4} \mathrm{Si}$ and $\mathrm{B}$ [Fig. S4(e) [59]]. In addition, 
some metastable structures of $\mathrm{BSi}, \mathrm{B}_{2} \mathrm{Si}, \mathrm{B}_{3} \mathrm{Si}$, and $\mathrm{B}_{4} \mathrm{Si}$ are also shown in Fig. S6 [59], which also presents similar structural characteristics to the predicted stable high-pressure structures.

For Si-rich compounds, the predicted $I 4 / m \mathrm{BSi}_{2}$ [Fig. 2(g)] is similar to the well-known $\mathrm{CuAl}_{2}$-type arrangement (some semi-transition-metal borides such as $\mathrm{Ti}_{2} \mathrm{~B}, \mathrm{Ta}_{2} \mathrm{~B}$, and $\mathrm{Mo}_{2} \mathrm{~B}$ also show the $I 4 / m$ symmetry [66]), in which $\mathrm{B}$ atoms form linear chains, where each B is surrounded by eight $\mathrm{Si}$ atoms. $\mathrm{BSi}_{3}$ was calculated to be unstable and dissociates into pure elements even at $400 \mathrm{GPa}$. The predicted $P-1$ and $P 2_{1} / m-2$ phases of $\mathrm{BSi}_{3}$ (Fig. S7 [59]) are energetically more stable than the $C 2 / m$ phase predicted by Wang et al. [29] in the corresponding pressure range.

The electron localization functions (ELFs) were calculated in order to understand the nature of the chemical bonds. As illustrated in Fig. S8 [59], high ELF values are distributed between $\mathrm{B}$ atoms as well as between $\mathrm{B}$ and $\mathrm{Si}$ atoms, indicating the presence of $\mathrm{B}-\mathrm{B}$ and $\mathrm{B}-\mathrm{Si}$ covalent bonds. In both $R \overline{3} m$ and $P \overline{3} m 1 \mathrm{~B}_{2} \mathrm{Si}$, all $\mathrm{B}$ atoms are bonded to $\mathrm{Si}$ atoms. However, in the $P \overline{3} m 1 \mathrm{~B}_{3} \mathrm{Si}$ and $\mathrm{B}_{4} \mathrm{Si}$, with multiboron layers, those $\mathrm{B}$ atoms further located away from $\mathrm{Si}$ atoms are only bonded to other B atoms. Bader charge calculations [67] have also been performed to analyze the charge transfer between atoms (Table S1 [59]). At $1 \mathrm{~atm}$, each $\mathrm{Si}$ atom transfers 1.56 and 1.2 electrons to two $\mathrm{B}$ atoms in $R \overline{3} m$ and $P \overline{3} m 1 \quad \mathrm{~B}_{2} \mathrm{Si}$, respectively. With increasing $\mathrm{B}$ content, in $\mathrm{B}_{3} \mathrm{Si}$ and $\mathrm{B}_{4} \mathrm{Si}$, there are still only two $\mathrm{B}$ atoms accepting electrons from one $\mathrm{Si}$ atom; the remaining $\mathrm{B}$ atoms even act as electron donors, transferring electrons to the two $\mathrm{B}$ atoms bonded to the $\mathrm{Si}$ atom. These results are consistent with the ELF calculations presented above.

The calculated electronic band structures and density of states (DOS) of stable structures of B-rich compounds are shown in Fig. 3 and Fig. S9 [59]. Several bands crossing the Fermi level $\left(\mathrm{E}_{f}\right)$ reveal the metallic character of these structures at $1 \mathrm{~atm}$. Similar to $\mathrm{MgB}_{2}$, the $\mathrm{B} p$ orbits give main contributions to the electronic states near the $E_{f}$. Band projections onto $\mathrm{B} p_{x}, p_{y}$, and $p_{z}$ orbitals are also shown in the band structures of $P \overline{3} m 1 \quad \mathrm{~B}_{2} \mathrm{Si}, \mathrm{B}_{3} \mathrm{Si}$, and $\mathrm{B}_{4} \mathrm{Si}$. In $P \overline{3} m 1 \mathrm{~B}_{2} \mathrm{Si}$ [Fig. 3(a)], the flat bands along the $\Gamma-A$ direction and around $\Gamma$ point near the $E_{f}$ are mainly derived from $\mathrm{B} p_{x, y}$, while the steep bands crossing $E_{f}$ derived from B $p_{z}$. Moreover, along the $\Gamma-A$ direction the nearly cylindrical Fermi surfaces also appeared, which arise from $p_{x, y} \mathrm{~B}$ bands (Fig. S10 [59]). These characters in $P \overline{3} m 1 \mathrm{~B}_{2} \mathrm{Si}$ are similar to those in $\mathrm{MgB}_{2}$ [68], which might imply the superconductivity of $P \overline{3} m 1 \mathrm{~B}_{2} \mathrm{Si}$. On the other hand, the flat bands close to the $E_{f}$ corresponding to localized electronic states lead to a large DOS value at $E_{f}$, which might also favor an enhancement of the electron-phonon interaction. As we mentioned earlier, B atoms exist in the forms of wrinkled single and double layers in $P \overline{3} m 1 \quad \mathrm{~B}_{2} \mathrm{Si}$ and $\mathrm{B}_{4} \mathrm{Si}$, respectively, while both forms of $\mathrm{B}$ atoms are alternately arranged in $P \overline{3} m 1 \mathrm{~B}_{3} \mathrm{Si}$. Thus the band structure of $P \overline{3} \mathrm{~m} 1 \mathrm{~B}_{3} \mathrm{Si}$ [Fig. 3(c)] has similar characteristics to that of $P \overline{3} m 1 \mathrm{~B}_{2} \mathrm{Si}$ [Fig. 3(a)] and $\mathrm{B}_{4} \mathrm{Si}$ [Fig. 3(e)], respectively. Along the $\Gamma-A$ line and around $\Gamma$ point in the band structure of $P \overline{3} m 1 \mathrm{~B}_{3} \mathrm{Si}$, the $\mathrm{B} p_{x, y}$ dominated bands near $E_{f}$ are mainly related to the single-layer $\mathrm{B}$ atoms, while the bands around 2 and $-2 \mathrm{eV}$ are relevant to the double-layer $\mathrm{B}$ atoms.
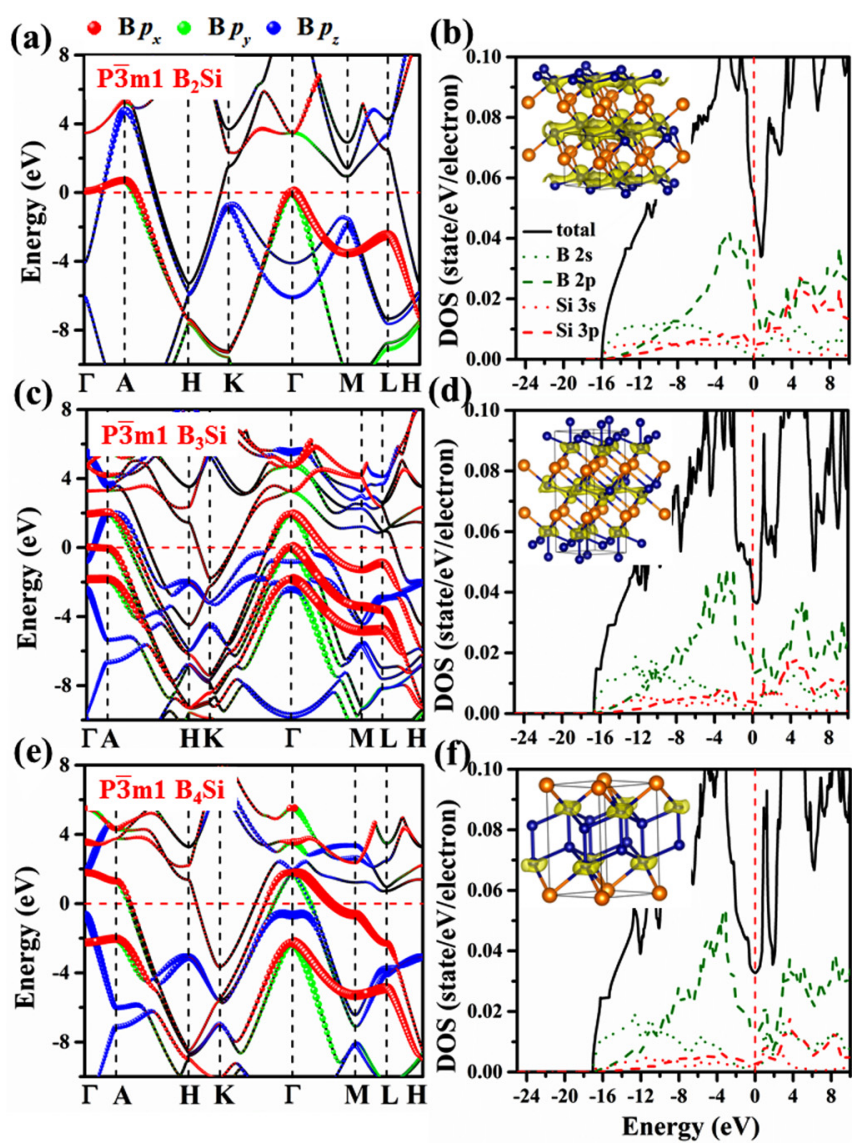

FIG. 3. Calculated electronic band structures and density of states for (a), (b) $P \overline{3} m 1 \mathrm{~B}_{2} \mathrm{Si}$, (c), (d) $P \overline{3} m 1 \mathrm{~B}_{3} \mathrm{Si}$, and (e), (f) $P \overline{3} m 1$ $\mathrm{B}_{4} \mathrm{Si}$ at $1 \mathrm{~atm}$. The bands projected onto $\mathrm{B} p_{x}, p_{y}$ and $p_{z}$ orbitals are displayed in the band structures (the radii of the circles are proportional to the weights of the corresponding orbitals). The insets in (b), (d), and (f) show the distributions of partial charge density with isosurface of 0.018 for states around the Fermi level.

Moreover, $P \overline{3} m 1 \mathrm{~B}_{2} \mathrm{Si}$ shows the highest DOS at Fermi level, while the lowest one corresponds to $P \overline{3} m 1 \mathrm{~B}_{4} \mathrm{Si}$. From $\mathrm{B}_{2} \mathrm{Si}$ to $\mathrm{B}_{4} \mathrm{Si}$ the position of the Fermi level gradually slides down to the valley of the pseudogap.

The insets in the right panels of Fig. 3 show the calculated partial charge densities with isosurface of 0.018 of states around the Fermi level. It can be seen that the calculated charge densities are mainly distributed around $\mathrm{B}$ atoms. In $P \overline{3} m 1 \mathrm{~B}_{2} \mathrm{Si}$, B-B $\sigma$-bond orbitals form conducting channels for valence electrons. Charge densities of states around the Fermi level come from connected electronic channels in the graphenelike B layer. With increasing the number of B layers in $\mathrm{B}_{3} \mathrm{Si}$ and $\mathrm{B}_{4} \mathrm{Si}$, the electronic channels are gradually destroyed, and charge densities are mainly distributed around the $\mathrm{B}$ atoms bonded with $\mathrm{Si}$ atoms, which suggests the charge transfer from Si to B atoms. Moreover, the other $\mathrm{B}$ atoms that are not bonded with $\mathrm{Si}$ atoms lose electrons and act as donors. According to the calculated partial charge densities, the metallicity of $P \overline{3} m 1 \mathrm{~B}_{2} \mathrm{Si}$ is stronger than that of $P \overline{3} m 1 \quad \mathrm{~B}_{3} \mathrm{Si}$ and $\mathrm{B}_{4} \mathrm{Si}$, which is consistent with the calculated DOS. Additionally, the electronic properties of 

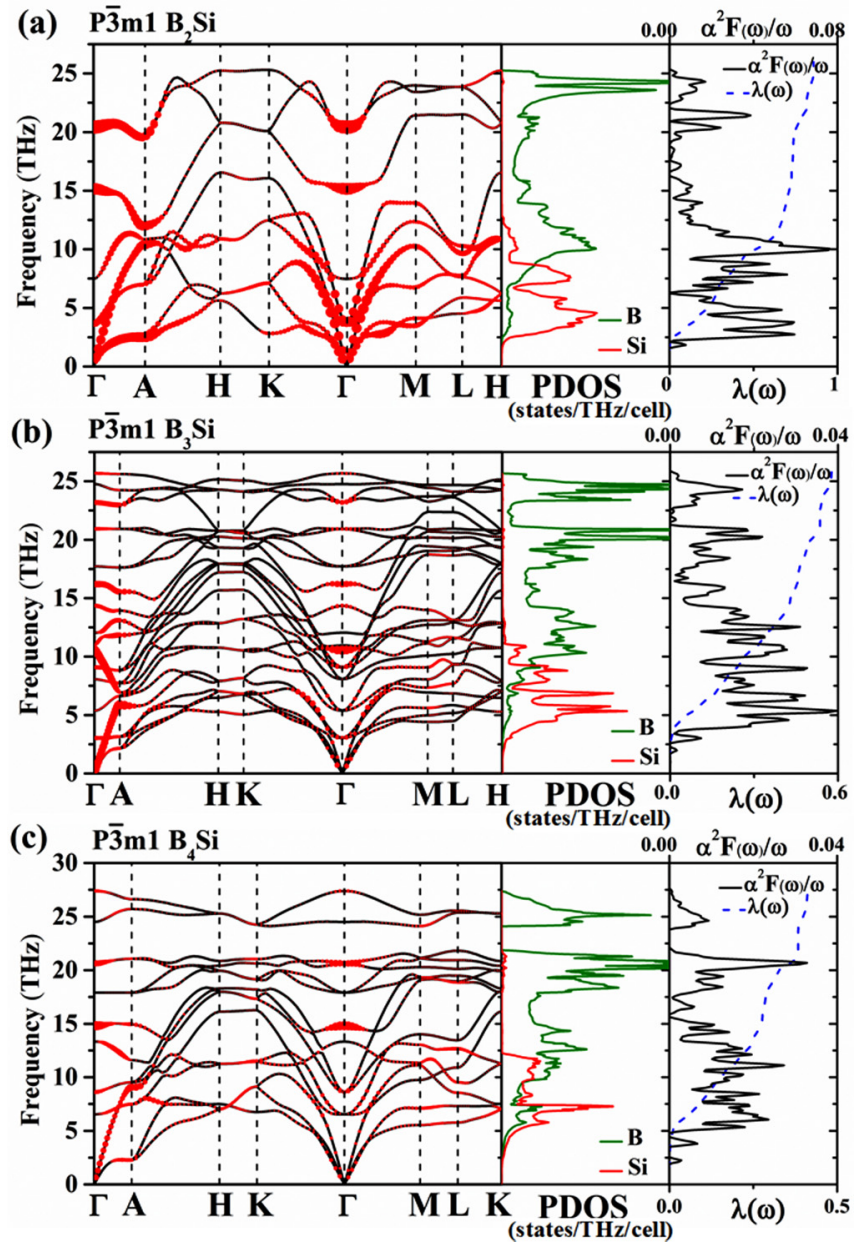

FIG. 4. Calculated phonon dispersion curves (the area of the red circles is proportional to the electron-phonon coupling strength), projected phonon density of states (PDOS), the Eliashberg phonon spectral function $\alpha^{2} F(\omega) / \omega$ and the electron-phonon integral $\lambda(\omega)$ of (a) $P \overline{3} m 1 \quad \mathrm{~B}_{2} \mathrm{Si}$, (b) $P \overline{3} m 1 \quad \mathrm{~B}_{3} \mathrm{Si}$, and (c) $P \overline{3} m 1 \quad \mathrm{~B}_{4} \mathrm{Si}$ at $1 \mathrm{~atm}$.

the predicted low-pressure structures of B-rich compounds and high-pressure structures of $\mathrm{BSi}$ and $\mathrm{BSi}_{2}$ are shown in Fig. S11 [59]. $\beta-\mathrm{B}_{3} \mathrm{Si}$ was estimated to be a semiconductor with an indirect band gap of about $1.3 \mathrm{eV}$, which is consistent with previous study [14]. The high-pressure phases of BSi and $\mathrm{BSi}_{2}$ are metallic.

As mentioned above, the predicted high-pressure structures of B-rich compounds are metastable at $1 \mathrm{~atm}$, with structural characteristics similar to $\mathrm{MgB}_{2}$. Having in mind that $\mathrm{MgB}_{2}$ is a good superconductor, with a $T_{c}$ of $39 \mathrm{~K}$, we thus performed phonon dispersion, projected phonon DOS, Eliashberg spectral function $\alpha^{2} F(\omega) / \omega$, and its integral $\lambda(\omega)$ to further explore the superconducting properties of the predicted metallic phases of $\mathrm{B}_{2} \mathrm{Si}, \mathrm{B}_{3} \mathrm{Si}$, and $\mathrm{B}_{4} \mathrm{Si}$ at 1 atm (Fig. 4). For $\mathrm{B}_{2} \mathrm{Si}$, the calculated phonon DOS can be separated into two regions. The low-frequency vibrations $(0-12.5 \mathrm{THz})$ are associated with both $\mathrm{Si}$ and $\mathrm{B}$ atoms and high-frequency modes are only related to $\mathrm{B}$ atoms. The calculated logarithmic average frequency $\omega_{\log }$ is $393 \mathrm{~K}$ and the EPC parameter $\lambda$ is 0.86 . The main contribution to the EPC comes from the low-

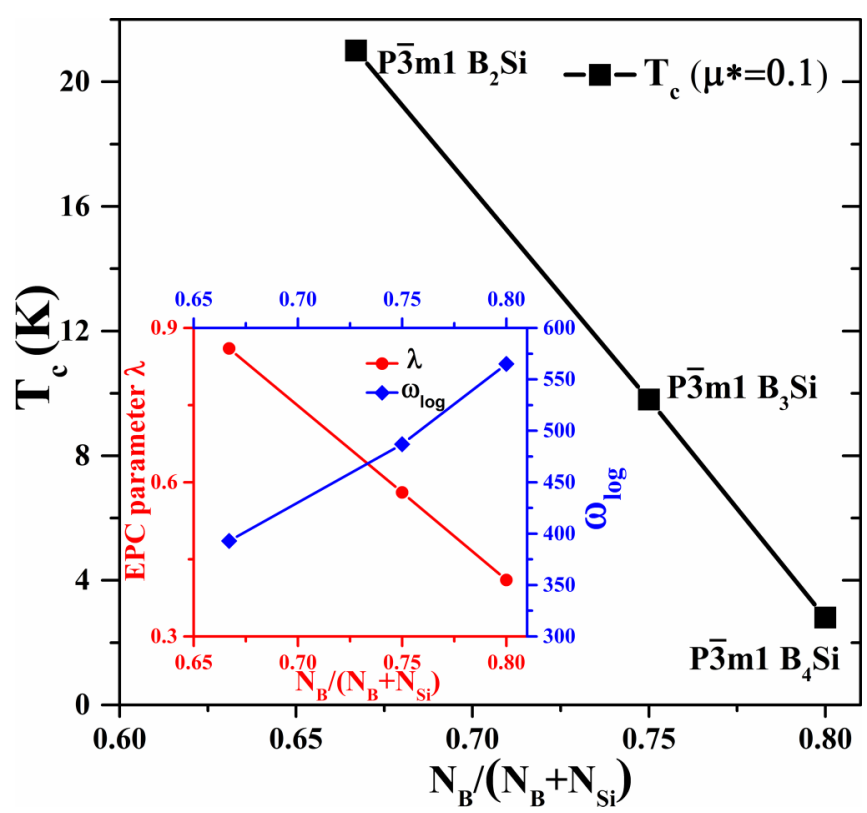

FIG. 5. Estimated $T_{c}$ values of similar structures $P \overline{3} m 1 \quad \mathrm{~B}_{2} \mathrm{Si}$, $P \overline{3} m 1 \mathrm{~B}_{3} \mathrm{Si}$, and $P \overline{3} m 1 \mathrm{~B}_{4} \mathrm{Si}$ at $1 \mathrm{~atm}$. The inset shows the evolution of the corresponding electron-phonon coupling parameter $\lambda$ and logarithmic average frequency $\omega_{\log }$ with increasing the $\mathrm{B}$ content from $\mathrm{B}_{2} \mathrm{Si}$ to $\mathrm{B}_{4} \mathrm{Si}$.

frequency modes, with $78 \%$ of the total $\lambda$. To further explore the contribution of different phonon modes, red circles with the area proportional to the EPC strength are also plotted in the phonon dispersion curves. All the phonon modes along $\Gamma-A$ direction have large contributions to the EPC, as well as the soft modes around $\Gamma$ point. The cases in $P \overline{3} m 1$ $\mathrm{B}_{3} \mathrm{Si}$ and $\mathrm{B}_{4} \mathrm{Si}$ are similar to that of $\mathrm{B}_{2} \mathrm{Si}$. However, the calculated EPC parameter $\lambda$ decreases from 0.86 in $P \overline{3} m 1$ $\mathrm{B}_{2} \mathrm{Si}$ to 0.68 and 0.41 in $P \overline{3} m 1 \mathrm{~B}_{3} \mathrm{Si}$ and $\mathrm{B}_{4} \mathrm{Si}$, respectively (Fig. 5), which mainly comes from the decreased contribution of the low-frequency modes from $78 \%, 69 \%$ to $56 \%$, respectively.

The $T_{c}$ values of these predicted phases were calculated by using the Allen-Dynes modified McMillan equation [69]. As shown in Fig. 5, the estimated $T_{c}$ of $P \overline{3} m 1 \mathrm{~B}_{2} \mathrm{Si}, \mathrm{B}_{3} \mathrm{Si}$, and $\mathrm{B}_{4} \mathrm{Si}$ at $1 \mathrm{~atm}$ is $21-17.5,9.8-6.8$, and $2.8-1.3 \mathrm{~K}$ by applying a Coulomb pseudopotential $\mu^{*}$ of $0.1-0.13$, respectively, where $T_{c}$ decreases with increasing B content. The trends of $\omega_{\log }$ and $\lambda$ as a function of the B content are shown in the inset of Fig. 5. The calculated $\omega_{\log }$ increases with increasing B content, which can be attributed to the presence of more and stronger B-B bonds. However, $\lambda$ presents an opposite evolution to $\omega_{\log }$. In addition, $R \overline{3} m \mathrm{~B}_{2} \mathrm{Si}$ and Pnma $\mathrm{BSi}$ are also estimated to superconduct with $T_{c}$ of 7.7 and $2.4 \mathrm{~K}$ at $1 \mathrm{~atm}$ and $250 \mathrm{GPa}$, respectively (Fig. S12 [59]). Our results show that most of the predicted high-pressure phases of $\mathrm{B}-\mathrm{Si}$ compounds are potential superconductors. $P \overline{3} m 1 \mathrm{~B}_{2} \mathrm{Si}$ shows the highest $T_{c}$ value of $21 \mathrm{~K}$ at $1 \mathrm{~atm}$, which is much higher than the experimentally observed $T_{c}$ of $0.56 \mathrm{~K}$ [19] in boron doped diamond-type silicon.

As we know, $\mathrm{MgB}_{2}$ is a prototypical anisotropic superconductor. Considering the similarity between $\mathrm{B}_{2} \mathrm{Si}$ and $\mathrm{MgB}_{2}$, 
(a)
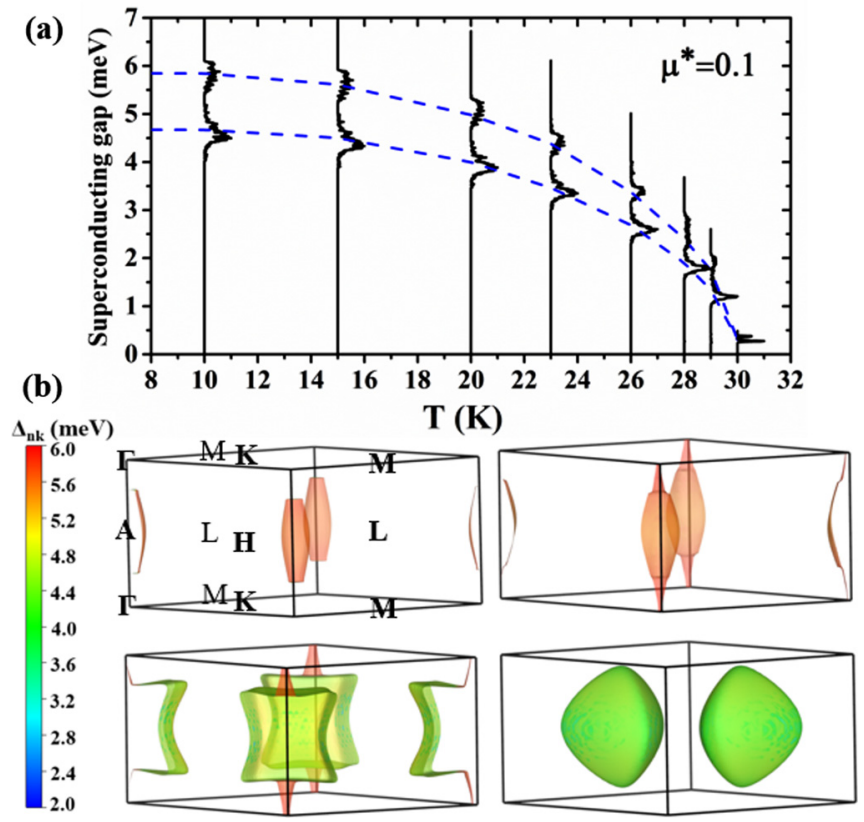

FIG. 6. (a) Calculated anisotropic superconducting gap of $P \overline{3} m 1 \mathrm{~B}_{2} \mathrm{Si}$ at $1 \mathrm{~atm}$ on the Fermi surface as a function of temperature. (b) The superconducting energy gap of $\mathrm{B}_{2} \mathrm{Si}$, calculated at $10 \mathrm{~K}$, mapped on the four Fermi surface sheets.

the superconducting anisotropy of $P \overline{3} m 1 \mathrm{~B}_{2} \mathrm{Si}$ was also investigated by combining the fully anisotropic Migdal-Eliashberg theory [52,53] with the electron-phonon interpolation based on maximally localized Wannier functions [57]. Figure 6(a) shows the calculated superconducting energy gap $\Delta_{n k}$ as a function of temperature with $\mu^{*}$ of 0.1 . One can clearly see that the $P \overline{3} m 1 \mathrm{~B}_{2}$ Si displays an anisotropic superconductivity with a two-gap nature. From the momentum-resolved superconducting gap $\Delta_{n k}$ on the Fermi surface at $10 \mathrm{~K}$ [Fig. 6(b)], we find that the higher $\Delta_{n k}$ values are distributed on the two Fermi surface sheets along the $\Gamma-A-\Gamma$ line associated to the $p_{x, y}$ orbitals of boron, while the lower $\Delta_{n k}$ values are mapped on the remaining Fermi surface sheets. In comparison, the energy difference of $1.2 \mathrm{meV}$ between two average $\Delta_{n k}$ values in $\mathrm{B}_{2} \mathrm{Si}$ is much smaller than that of $6.5 \mathrm{meV}$ in $\mathrm{MgB}_{2}$ at $10 \mathrm{~K}$ [56], indicating that $\mathrm{B}_{2} \mathrm{Si}$ is less anisotropic. In $\mathrm{MgB}_{2}$, there are not only strong covalent $\mathrm{B}-\mathrm{B}$ bonds in the graphenelike planes, but also ionic bonds between $\mathrm{Mg}$ and $\mathrm{B}$ atoms. Two degenerate $\sigma$-bond bands associated to B atoms are highly coupled with the $E_{2 g}$ phonon mode, which is mainly associated with the B atomic motion [70]. However, in the predicted $\mathrm{B}_{2} \mathrm{Si}$, the interplane $\mathrm{B}-\mathrm{Si}$ bonds are also strongly covalent. The bands near the Fermi level mainly associated to B orbitals are not only coupled with B-related phonon modes, but also with Si-related ones. As temperature increases, the superconducting gap will decrease. The critical temperature
$T_{c}$ can be estimated when the superconducting gap value vanishes. As shown in Fig. 6(a), the critical temperature $T_{c}$ for $P \overline{3} m 1 \mathrm{~B}_{2} \mathrm{Si}$ is $30 \mathrm{~K}$, which is higher than that of $21 \mathrm{~K}$ derived with the Allen-Dynes formula.

\section{CONCLUSIONS}

In summary, we have systematically explored the crystal structures and novel properties of B-Si compounds under pressure. At $1 \mathrm{~atm}$, we reproduced the experimentally observed ordered structure $\beta-\mathrm{B}_{3} \mathrm{Si}$ and also successfully predicted a series of structures containing $\mathrm{B}_{12}$ units for $\mathrm{B}_{6} \mathrm{Si}$ and $\mathrm{B}_{4} \mathrm{Si}$, which are similar to the experimental ones. Upon compression, new compositions, $\mathrm{B}_{2} \mathrm{Si}, \mathrm{BSi}$, and $\mathrm{BSi}_{2}$, become gradually stable, indicating that pressure promotes the formation of $\mathrm{B}-\mathrm{Si}$ compounds with high silicon content. On the B-rich side, from $\mathrm{B}_{2} \mathrm{Si}$ to $\mathrm{B}_{6} \mathrm{Si}$, all the predicted stable high-pressure structures contain buckled graphenelike boron layers and the number of boron layers between adjacent silicon layers increases with increasing the boron content. Phonon calculations show these structures are dynamically stable, so that they might be preserved as metastable phases at $1 \mathrm{~atm}$. Electronic band structure calculations show that all the stable high-pressure phases are metallic. Moreover, electron-phonon coupling calculations indicate that all the retained high-pressure phases of B-rich compounds are superconductors at $1 \mathrm{~atm} . P \overline{3} m 1 \mathrm{~B}_{2} \mathrm{Si}$ has the highest $T_{c}$ value of $21 \mathrm{~K}$ at $1 \mathrm{~atm}$, which is much higher than that of the observed one in B doped diamond-type Si. Within the fully anisotropic Migdal-Eliashberg formalism, our calculations suggest that $P \overline{3} m 1 \mathrm{~B}_{2} \mathrm{Si}$ is a two-gap anisotropic superconductor and the estimated $T_{c}$ might reach up to $30 \mathrm{~K}$ at $1 \mathrm{~atm}$. Our studies not only enrich the phase diagram of the B-Si system but also may stimulate experimental exploration of superconductivity in B-Si compounds. Moreover, the B-Si system will be helpful for further understanding structural and physical properties of boron-rich compounds under pressure.

\section{ACKNOWLEDGMENTS}

The work was supported by Fostering Program of Major Research Plan of NSFC (91963115), National Key R \& D Program of China (2018YFA0703400), National Natural Science Foundation of China (No. 51732010, 11674176, 11874224), Funding Program for Recruited Oversea Scholars of Hebei Province (Grant No. CL201729), and the Ph.D. Foundation by Yanshan University (Grant No. B970). A.B. acknowledges financial support from the Spanish Ministry of Economy and Competitiveness (Grant No. FIS2016-76617-P) and the Department of Education, Universities and Research of the Basque Government and the University of the Basque Country (Grant No. IT756-13).
[1] B. Albert and H. Hillebrecht, Angew. Chem. Int. Ed. 48, 8640 (2009).
[2] G. Akopov, M. T. Yeung, and R. B. Kaner, Adv. Mater. 29, 1604506 (2017). 
[3] A. V. D. Geest and A. Kolmogorov, Calphad 46, 184 (2014).

[4] J. Nagamatsu, N. Nakagawa, T. Muranaka, Y. Zenitani, and J. Akimitsu, Nature (London) 410, 63 (2001).

[5] H. Gou, N. Dubrovinskaia, E. Bykova, A. A. Tsirlin, D. Kasinathan, W. Schnelle, A. Richter, M. Merlini, M. Hanfland, A. M. Abakumov et al., Phys. Rev. Lett. 111, 157002 (2013).

[6] H. Rosner, A. Kitaigorodsky, and W. E. Pickett, Phys. Rev. Lett. 88, 127001 (2002).

[7] A. N. Kolmogorov, S. Shah, E. R. Margine, A. F. Bialon, T. Hammerschmidt, and R. Drautz, Phys. Rev. Lett. 105, 217003 (2010).

[8] H.-Y. Chung, M. B. Weinberger, J. B. Levine, A. Kavner, J.-M. Yang, S. H. Tolbert, and R. B. Kaner, Science 316, 436 (2007)

[9] M. Mukaida, T. Goto, and T. Hirai, Mater. Manuf. Process. 7, 625 (1992).

[10] B. Armas, C. Combescure, J. Dusseau, T. Lepetre, J. Robert, and B. Pistoulet, J. Less Common Met. 47, 135 (1976).

[11] C. Wood, Rep. Prog. Phys. 51, 459 (1988).

[12] T. Lundstrom, Pure Appl. Chem. 57, 1383 (1985).

[13] H. Rizzo, B. Weber, and M. Schwartz, J. Am. Ceram. Soc. 43, 497 (1960).

[14] J. R. Salvador, D. Bilc, S. Mahanti, and M. G. Kanatzidis, Angew. Chem. Int. Ed. 42, 1929 (2003).

[15] Y. Imai, M. Mukaida, M. Ueda, and A. Watanabe, J. Alloy Compd. 347, 244 (2002).

[16] E. Bustarret, C. Marcenat, P. Achatz, J. Kačmarčik, F. Lévy, A. Huxley, L. Ortéga, E. Bourgeois, X. Blase, D. Débarre et al., Nature (London) 444, 465 (2006).

[17] K. Kádas, L. Vitos, and R. Ahuja, Appl. Phys. Lett. 92, 052505 (2008).

[18] X. Blase, E. Bustarret, C. Chapelier, T. Klein, and C. Marcenat, Nat. Mater. 8, 375 (2009).

[19] C. Marcenat, J. Kačmarčík, R. Piquerel, P. Achatz, G. Prudon, C. Dubois, B. Gautier, J. C. Dupuy, E. Bustarret, L. Ortega, T. Klein, J. Boulmer, T. Kociniewski, and D. Debarre, Phys. Rev. B 81, 020501(R) (2010).

[20] H. Moissan and A. Stock, C. R. Acad. Sci. (Paris) 131, 139 (1900).

[21] R. Olesinski and G. Abbaschian, Bull. Alloy Phase Diagr. 5, 478 (1984).

[22] B. Magnusson and C. Brosset, Acta Chem. Scand. 16, 449 (1962).

[23] T. Aselage, J. Mater. Res. 13, 1786 (1998).

[24] R. Adamsky, Acta Crystallogr. 11, 744 (1958).

[25] C. F. Cline, J. Electrochem. Soc. 106, 322 (1959).

[26] R. Geise, Jr., J. Economy, and V. Matkovich, Z. Kristallogr. 122, 144 (1965).

[27] M. Vlasse and J. Viala, J. Solid State Chem. 37, 181 (1981).

[28] J. Viala and J. Bouix, J. Less Common Met. 71, 195 (1980).

[29] J. Wang, G. Sun, P. Kong, W. Sun, C. Lu, F. Peng, and X. Kuang, Phys. Chem. Chem. Phys. 19, 16206 (2017).

[30] D. T. T. Mai, L. V. Duong, T. B. Tai, and M. T. Nguyen, J. Phys. Chem. A 120, 3623 (2016).

[31] T. B. Tai, P. Kadłubański, S. Roszak, D. Majumdar, J. Leszczynski, and M. T. Nguyen, Chem. Phys. Chem. 12, 2948 (2011).
[32] X. Tan, F. Li, and Z. Chen, J. Phys. Chem. C 118, 25825 (2014).

[33] X. Tan, C. R. Cabrera, and Z. Chen, J. Phys. Chem. C 118, 25836 (2014).

[34] Y. Ding and Y. Wang, J. Phys. Chem. C 117, 18266 (2013).

[35] Y. Wang, J. Lv, L. Zhu, and Y. Ma, Phys. Rev. B 82, 094116 (2010).

[36] J. Lv, Y. Wang, L. Zhu, and Y. Ma, J. Chem. Phys. 137, 084104 (2012).

[37] Y. Wang, J. Lv, L. Zhu, and Y. Ma, Comput. Phys. Commun. 183, 2063 (2012).

[38] L. Zhu, H. Liu, C. J. Pickard, G. Zou, and Y. Ma, Nat. Chem. 6 , 644 (2014).

[39] X. Liang, A. Bergara, L. Wang, B. Wen, Z. Zhao, X.-F. Zhou, J. He, G. Gao, and Y. Tian, Phys. Rev. B 99, 100505(R) (2019).

[40] L. Wang, F. Tian, X. Liang, Y. Fu, X. Mu, J. Sun, X.-F. Zhou, K. Luo, Y. Zhang, Z. Zhao et al., Phys. Rev. B 99, 174104 (2019).

[41] L. Zhu, H. Wang, Y. Wang, J. Lv, Y. Ma, Q. Cui, Y. Ma, and G. Zou, Phys. Rev. Lett. 106, 145501 (2011).

[42] J. Lv, Y. Wang, L. Zhu, and Y. Ma, Phys. Rev. Lett. 106, 015503 (2011).

[43] J. Lin, S. Zhang, W. Guan, G. Yang, and Y. Ma, J. Am. Chem. Soc. 140, 9545 (2018).

[44] Z. Zhao, S. Zhang, T. Yu, H. Xu, A. Bergara, and G. Yang, Phys. Rev. Lett. 122, 097002 (2019).

[45] G. Kresse and J. Furthmüller, Phy. Rev. B 54, 11169 (1996).

[46] J. P. Perdew, K. Burke, and M. Ernzerhof, Phys. Rev. Lett. 77, 3865 (1996).

[47] P. E. Blöchl, Phys. Rev. B 50, 17953 (1994).

[48] H. J. Monkhorst and J. D. Pack, Phys. Rev. B 13, 5188 (1976).

[49] K. Parlinski, Z. Q. Li, and Y. Kawazoe, Phys. Rev. Lett. 78, 4063 (1997).

[50] A. Togo, F. Oba, and I. Tanaka, Phys. Rev. B 78, 134106 (2008).

[51] P. Giannozzi, S. Baroni, N. Bonini, M. Calandra, R. Car, C. Cavazzoni, D. Ceresoli, G. L. Chiarotti, M. Cococcioni, I. Dabo et al., J. Phys.: Condens. Matter 21, 395502 (2009).

[52] P. B. Allen and B. Mitrović, Solid State Physics (Elsevier, Amsterdam, 1983), Vol. 37, pp. 1-92.

[53] E. R. Margine and F. Giustino, Phys. Rev. B 87, 024505 (2013).

[54] F. Giustino, M. L. Cohen, and S. G. Louie, Phys. Rev. B 76, 165108 (2007)

[55] J. Noffsinger, F. Giustino, B. D. Malone, C.-H. Park, S. G. Louie, and M. L. Cohen, Comput. Phys. Commun. 181, 2140 (2010).

[56] S. Poncé, E. R. Margine, C. Verdi, and F. Giustino, Comput. Phys. Commun. 209, 116 (2016).

[57] N. Marzari, A. A. Mostofi, J. R. Yates, I. Souza, and D. Vanderbilt, Rev. Mod. Phys. 84, 1419 (2012).

[58] A. A. Mostofi, J. R. Yates, Y.-S. Lee, I. Souza, D. Vanderbilt, and N. Marzari, Comput. Phys. Commun. 178, 685 (2008).

[59] See Supplemental Material at http://link.aps.org/supplemental/ 10.1103/PhysRevB.101.014112 for the structural comparison and energy barrier between $\beta$ - $\mathrm{B}_{3} \mathrm{Si}$ (Imma) and predicted $P_{m m a B_{3}} \mathrm{Si}$ at $1 \mathrm{~atm}$; the predicted metastable structures for $\mathrm{B}_{4} \mathrm{Si}$ and $\mathrm{B}_{6} \mathrm{Si}$ at $1 \mathrm{~atm}$; the calculated enthalpy curves for different $\mathrm{B}$-Si compounds as a function of pressure, with respect to the elements and other stoichiometries; the predicted metastable 
structures for B-Si compounds under pressure; the calculated ELFs and band structures of some predicted stable structures of B-Si compounds; the calculated band structure and DOS for $R \overline{3} m \mathrm{~B}_{2} \mathrm{Si}$ at $1 \mathrm{~atm}$; the Fermi surface of $P \overline{3} m 1 \mathrm{~B}_{2} \mathrm{Si}$ at $1 \mathrm{~atm}$; the superconductivity for $R \overline{3} m \mathrm{~B}_{2} \mathrm{Si}$ and Pnma BSi at 1 atm and $250 \mathrm{GPa}$, respectively; phonon spectra of the various structures; the Bader charge analysis for predicted stable B-rich phases at $1 \mathrm{~atm}$; the calculated $T_{c}$ for various predicted stable phases at different pressures; detail structural information of predicted B-Si compounds at selected pressures.

[60] I. Higashi, M. Kobayashi, J. Bernhard, C. Brodhag, and F. Thévenot, AIP Conf. Proc. 231, 201 (1991).

[61] N. Vojteer, M. Schroeder, C. Roehr, and H. Hillebrecht, Chem. Eur. J. 14, 7331 (2008).

[62] Y. Yao and D. D. Klug, Phys. Rev. B 85, 214122 (2012).
[63] A. R. Oganov, J. Chen, C. Gatti, Y. Ma, Y. Ma, C. W. Glass, Z. Liu, T. Yu, O. O. Kurakevych, and V. L. Solozhenko, Nature (London) 457, 863 (2009).

[64] D. Li, K. Bao, F. Tian, X. Jin, D. Duan, Z. He, B. Liu, and T. Cui, RSC Adv. 4, 203 (2014).

[65] J. Kunstmann and A. Quandt, Phys. Rev. B 74, 035413 (2006).

[66] M. Wang, C. Liu, M. Wen, Q. Li, and Y. Ma, Phys. Chem. Chem. Phys. 19, 31592 (2017).

[67] R. Bader, Atoms in Molecules: A Quantum Theory (Oxford University Press, Oxford, 1994).

[68] J. Kortus, I. I. Mazin, K. D. Belashchenko, V. P. Antropov, and L. L. Boyer, Phys. Rev. Lett. 86, 4656 (2001).

[69] P. B. Allen and R. Dynes, Phys. Rev. B 12, 905 (1975).

[70] Y. Kong, O. V. Dolgov, O. Jepsen, and O. K. Andersen, Phys. Rev. B 64, 020501(R) (2001). 